\title{
Using Eye-Tracking and Click-Stream Data to Design Adaptive Training of Children's Inhibitory Control in a Maths and Science Game
}

\author{
Andrea Gauthier ${ }^{1(\otimes)}\left(\mathbb{D}\right.$, Kaśka Porayska-Pomsta ${ }^{1}$ (D), Denis Mareschal $^{2}$ (D), \\ and The UnLocke Project Team \\ 1 Knowledge Lab, Institute of Education, University College London, \\ London WC1N 3QS, UK \\ \{andrea.gauthier, k. porayska-pomsta\}@ucl.ac.uk \\ 2 Department of Psychological Sciences, Birkbeck College University of London, \\ London WC1E 7HX, UK \\ d.mareschal@bbk.ac.uk
}

\begin{abstract}
Computerised educational neuroscience interventions that train within-domain inhibitory control (IC) can improve children's counterintuitive reasoning. However, the HCI or adaptive design of such environments often receive less attention. Eye-tracking and click data were used to compare four versions of an IC-training game in terms of their HCI design and potential for supporting adaptive feedback. Our results provide insights for developing an adaptive system to scaffold pupils' transition towards using IC in un-cued, self-regulated scenarios.
\end{abstract}

Keywords: Inhibitory control - Counterintuitive reasoning •

Educational neuroscience $\cdot$ Game-based learning $\cdot$ Adaptive support

\section{Introduction}

Inhibitory control (IC) belongs to a set of cognitive skills, known as "executive functions", that are foundational to self-regulated learning (SRL) $[1,9,18]$. IC is key to maths and science learning where inhibition of pre-existing beliefs or superficial perceptions is necessary for learning and applying counterintuitive knowledge $[7,13,14]$. There is a keen interest in understanding IC as a component of SRL and in applying Educational Neuroscience (EdN) insights to educational practice. While it is standard to computerise EdN interventions, many of these ignore key principles and methods at the intersection of HCI and AIED. We argue that such principles may be critical to a successful delivery of EdN interventions.

We present work conducted as part of an EdN-focused project (funded by the Wellcome Trust and Education Endowment Foundation), in which we developed a trivia game-based environment for children to train IC skills through exercising "stop and think" behaviours on counterintuitive concepts in science 
and maths [16]. Our work examines the relationship between the delivery of diverse visual prompts, their interference with in-game cognitive tasks, and how such interference can be remedied adaptively to promote IC. As such, this work explicitly bridges EdN, HCI and AIED research.

\section{Stop \& Think IC-Training Environment}

"Stop \& Think" (SET ) is a trivia game-based learning environment that gives children, aged 7-10, structured opportunities to exercise IC on counterintuitive maths and science concepts [16]. The game has two modes: (1) TV-trivia show mode where a host character presents quiz questions, while three virtual contestants articulate the reasoning behind their answers through speech bubbles; (2) active engagement mode that trains IC by having pupils pause for a few seconds (henceforth-the SET mechanic) before allowing them to answer each question, to encourage them to suppress intuitive thinking and adopt counterintuitive (but correct) concepts. A teacher-led version of this non-adaptive software was evaluated with 6672 children from 89 schools in England. Children played S\&T as a whole class for $12 \mathrm{~min}$., $3 \times /$ week, for 10 weeks, with a teacher acting as facilitator. The study revealed that training IC in this way improved counterintuitive reasoning and standardised exam scores [16]. We now investigate how the following HCI features might enhance S\&T's IC training in a SRL scenario:

1. Mandatory interaction: indication of readiness. In line with the theory of planned behaviour [3], an interaction mechanic where pupils must indicate their readiness to answer, may promote IC through metacognitive awareness.

2. Pre-attentive cues: motion and colour. Pre-attentive cues are processed by our visual systems prior to conscious attention to guide gaze [15]. Persistent motion may consistently grab attention $[4,15]$. Colour has culturally relevant meanings $[15,17]$ that might guide attention, e.g. through the traffic light metaphor for "stop and go" types of activities [10].

3. Game mechanics: reward systems. Tangible rewards relay performance information, which may promote IC and metacognitive competencies [8,12]. Penalties create a sense of risk and contextualise the value of rewards $[8,11]$, potentially assisting IC training by motivating pupils to stop and think longer to improve performance and earn greater rewards.

Four versions of S\&T implement the above features (Fig. 1): Condition A (baseline) uses pulsating motion of a "Stop \& Think" icon for four seconds during the S\&T mechanic. The screen is 'locked' during this time, then the icon disappears and the screen becomes interactive. Condition B builds on A, adding mandatory interaction before the pupil can submit their answer. After the foursecond thinking time, the pulsating icon is replaced by a button that reads "I'm ready to answer!". Once pressed, the screen becomes interactive. Condition $\mathbf{C}$ also includes the mandatory interaction, but uses colour instead of motion to prompt IC behaviour. The common analogy of traffic lights is used on the S\&T icon to represent (1) "Stop" (red) during question narration; (2) "Think" 

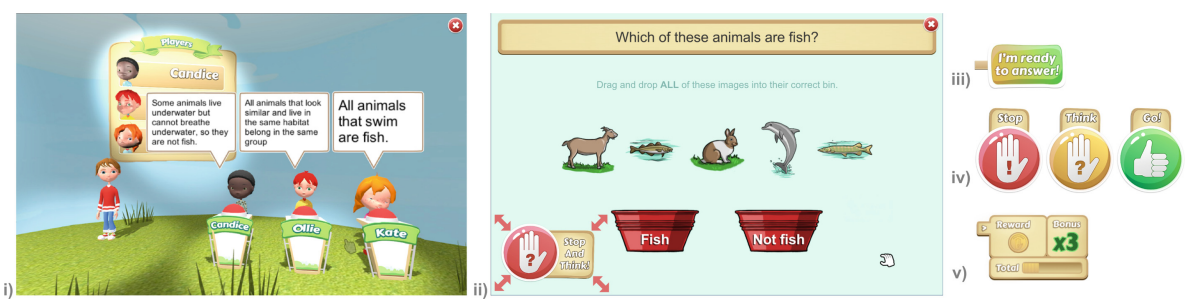

Fig. 1. (i) Game-show mode: contestants express their reasoning; (ii) Task mode: pulsating S\&T icon (Condition A-B); (iii) "I'm ready to answer" button (Conditions B-D); (iv) Traffic-light S\&T icons (Conditions C-D); (v) Scoreboard (Condition D). (color figure online)

(amber) during the S\&T mechanic (the "I'm ready" button pops beside the icon after four seconds); and (3) "Go" (green) when interaction is allowed (after the button is pressed). Condition $\mathbf{D}$ builds on $\mathrm{C}$ by integrating simple rewards (tokens for correct answers) and penalties (loss of bonus multiplier).

\section{$3 \quad$ Study Design and Results}

The efficacy of these four conditions at guiding IC was evaluated with children from two English primary schools. We used eye-tracking and click data to investigate which HCI design features encouraged the most consistent IC behaviour, then used these findings to suggest how the game might adapt to support IC.

Participants. 45 participants (19 girls and 26 boys), aged 7-8, were randomised across conditions (11 in Conditions A, B, D; 12 in Condition C).

Procedure. Each pupil's participation lasted about $30 \mathrm{~min}$, including an introduction, randomisation, eye-tracker calibration, 2-min video tutorial on how to play, and 12-min playtime. The eye-tracking station was set up on a laptop running Tobii Pro Studio with compact eye-tracker, in a quiet room.

Measures. Interaction (in-game performance, time spent stopping and thinking) and eye-tracking (fixation duration on areas of interest $-A o I$ ) data were analysed using Kruskal-Wallis $(K W)$, Wilcoxon signed rank $(W)$, Bonferroniadjested Mann-Whitney $U$ ( $U$, for pair-wise comparisons), and Spearman correlations $(r h o)$. In Tobii Studio, recordings were parsed into four scenes for each task attempt, based on phases of interaction relevant to IC training: (1) Question narration, (2) Mandatory S\&T time (first four seconds after narration), (3) Voluntary S\&T time (after first four seconds, before "I'm ready" button is pressed), and (4) Activity (while they complete the task). AoI were set for (i) answer-objects (image/number options), (ii) question text box, and (iii) S\&T icon. Fixation data were tallied for narration, mandatory, and voluntary S\&T phases; as Condition A did not have a voluntary S\&T phase, fixations were inevitably lower in this group, so they were excluded from fixation analyses. 
In-game Performance. Groups achieved similar scores in maths $(K W=$ $0.35, p=.951)$ and science $(K W=6.64, p=.084)$. Science scores were generally slightly higher than maths $(W=280.00, p=.081) .17 / 45$ participants earned a score of zero on the maths portion of the game (simple addition of fractions), compared to only four pupils on the science (fish vs. mammals).

Time Spent Stopping \& Thinking (S\&T Time). The average total S\&T time is the sum of "time to first click" during all four phases of the game, and averaged out over all science/maths tasks. There was no difference across conditions $(K W=5.29, p=.151)$ and no relationship between S\&T time and performance $(r h o=-0.10, p=.531)$ in maths tasks. Pupils struggled with maths, averaging $28.04 \mathrm{~s}(S D=21.01)$ of $\mathrm{S} \& \mathrm{~T}$ time. This is much longer than in science $(W=130.00, p<.001)$, where pupils averaged $15.72 \mathrm{~s}(S D=4.38)$, with a positive relationship between S\&T time and in-game performance $(r h o=0.48$, $p=.001)$. There was an effect of condition for science $(K W=11.71, p=.008)$ : Conditions B $(U=-15.27, p=.038), \mathrm{C}(U=-14.81, p=.041)$, and $\mathrm{D}(U=$ $-16.55, p=.019)$ had longer S\&T times than A.

Question Box AoI Fixations. Fixations were similar across groups in both maths $(K W=0.69, p=.709)$ and science tasks $(K W=1.38, p=.502)$.

Answer Objects AoI Fixations. There was an effect of condition in science $(K W=9.097, p=.011)$. Condition $\mathrm{C}$ averaged greater fixation duration $(U=$ $12.470, p=.008)$ than D. Overall, fixations in maths $(5.89 \mathrm{~s}, S D=7.92)$ and science $(5.63 \mathrm{~s}, S D=2.99)$ were similar $(W=635.00, p=.185)$.

S\&T Icon AoI Fixations. There were no differences between conditions in maths $(K W=3.50, p=.174)$ or science $(K W=3.50, p=.174)$.

\section{Discussion and Conclusions}

To inform the design of adaptive support in the context of domain-specific IC training, this study explored how HCI design decisions impacted children's ingame IC behaviours along with their performance. We found that (1) long S\&T times could either be indicative of IC behaviour (as seen in science tasks, in line with the literature $[5,16]$ ) or of confusion (as seen in maths); (2) mandatory interaction (i.e. "I'm ready" button) resulted in increased S\&T time, supporting the hypothesis that integrating a mechanic that allows pupils to indicate their readiness to answer may encourage planning of interactions [3]; (3) Symbolic colour and motion were both effective at promoting IC (B and $\mathrm{C}$ generated similar S\&T times and fixations on answer objects); and (4) Rewards/penalties did not impact behaviours, perhaps because the scoring mechanic did not provide feedback on the IC behaviour itself. Feedback is crucial for meta-learning [2], so future research should consider using more informative displays (like open learner models) to promote reflective thinking [6]. Our results also indicate three aspects of the game that might be adapted to support IC behaviours and aid children in transitioning to un-cued SRL scenarios: (i) mandatory S\&T time, 
based on the player's average S\&T time together with answer correctness as a measure to calibrate the optimal thinking time; (ii) difficulty of the content or level of support given to the pupil, but the system should differentiate between meaningful IC behaviour (as observed in science) and being 'lost' (maths); and (iii) visual cues (e.g. S\&T icon), which might be scaffolded away once pupils display consistent IC behaviour. This work examined the links between delivery of the diverse visual prompts, their interference with in-game cognitive tasks, and how such interference can be remedied adaptively within a SRL game to promote IC, to explicitly bridge EdN, HCI, and AIED research.

\section{References}

1. Allan, N.P., Hume, L.E., Allan, D.M., Farrington, A.L., Lonigan, C.J.: Relations between inhibitory control and the development of academic skills in preschool and kindergarten: a meta-analysis. Dev. Psychol. 50(10), 2368 (2019)

2. Askew, S., Lodge, C.: Gifts, ping-pong and loops? Linking feedback and learning. In: Askew, S. (ed.) Feedback For Learning, pp. 1-17 (2004)

3. Ajzen, I.: From intentions to actions: a theory of planned behavior. In: Kuhl, J., Beckmann, J. (eds.) Action Control. SSSSP, pp. 11-39. Springer, Heidelberg (1985). https://doi.org/10.1007/978-3-642-69746-3_2

4. Bartram, L., Ware, C., Calvert, T.: Moticons: detection, distraction and task. Int. J. Hum.? Comput. Stud. 58(5), 515-545 (2003)

5. Brookman-Byrne, A., Mareschal, D., Tolmie, A.K., Dumontheil, I.: Inhibitory control and counterintuitive science and maths reasoning in adolescence. PLoS ONE 13(6), 1-19 (2018)

6. Bull, S., Mangat, M., Mabbott, A., Abu Issa, A., Marsh, J.: Reactions to inspectable learner models: seven year olds to university students. In: Workshop on Learner Modelling for Reflection, International Conference on Artificial Intelligence in Education, AIED 2005, pp. 1-10 (2005)

7. Diamond, A., Lee, K.: Interventions shown to aid executive function development in children 4 to 12 years old. Science 333, 959-964 (2011)

8. Gee, J.P.: What Video Games Have to Teach Us About Learning and Literacy. Palgrave MacMillan, London (2007)

9. Jacob, R., Parkinson, J.: The potential for school-based interventions that target executive function to improve academic achievement: a review. Rev. Educ. Res. $\mathbf{8 5}(4), 512-552(2015)$

10. Jung, T., Huang, J., Eagan, L., Oldenburg, D.: Influence of school-based nutrition education program on healthy eating literacy and healthy food choice among primary school children. Dev. Psychol. 57(2), 67-81 (2019)

11. Juul, J.: Fear of failing? The many meanings of difficulty in video games. In: Wolf, M.J.P., Perron, B. (eds.) The Video Game Theory Reader, vol. 2, pp. 237-252 (2009). https://doi.org/10.1017/CBO9781107415324.004

12. Proulx, J.N., Romero, M., Arnab, S.: Learning mechanics and game mechanics under the perspective of self-determination theory to foster motivation in digital game based learning. Simul. Gaming 48(1), 81-97 (2017)

13. Rousselle, L., Palmers, E., Noel, M.P.: Magnitude comparison in preschoolers: what counts? Influence of perceptual variables. J. Exp. Child Psychol. 87(1), 57-84 (2004) 
14. Stavy, R., Babai, R.: Overcoming intuitive interference in mathematics: insights from behavioral, brain imaging and intervention studies. ZDM 42(6), 621-633 (2010)

15. Ware, C.: Information Visualization: Perception for Design. Elsevier Inc., Amsterdam (2013)

16. Wilkinson, H.R., et al.: Learning mechanics and game mechanics under the perspective of self-determination theory to foster motivation in digital game based learning. J. Cogn. Enhanc. 48(1), 81-97 (2019). https://doi.org/10.1007/s41465019-00161-4

17. Wong, B.: Points of view: color coding. Nat. Methods 7(8), 538 (2010)

18. Zelazo, P.D., Blair, C.B., Willoughby, M.T.: Executive function: implications for education. In: NCER (2016) 